\title{
Perfil de uso de medicamentos por idosos assistidos pelo Programa de Atenção ao Idoso (P.A.I.) da UNIJUÍ
}

Drug use profile of elderly patients attending the Elderly Care Program in UNIJUI

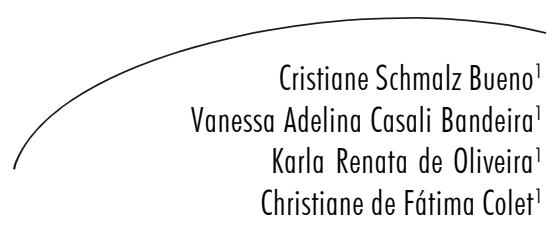

Resumo

O processo de envelhecimento é caracterizado por várias modificações no organismo, que podem causar alterações no efeito de certos medicamentos, tornando-os inapropriados para idosos por falta de eficácia terapêutica ou por apresentarem efeitos adversos superiores aos benefícios. O objetivo do estudo foi identificar os medicamentos utilizados pelos idosos assistidos pelo Programa de Atenção ao Idoso (P.A.I.) e investigar o uso de medicamentos potencialmente inapropriados nessa população. Realizou-se estudo quantitativo e descritivo, com coleta de dados secundários, a partir de prontuários/cadastros dos idosos atendidos pelo P.A.I. (Projeto de Extensão Universitária da UNIJUÍ). Entre os idosos cadastrados, 16 receberam e/ou estão recebendo acompanhamento farmacoterapêutico pela equipe da farmácia, sendo 11 mulheres, com idade média de 75,2 \pm 7,5 anos. Cada idoso recebe em média 7,3 \pm 3,3 medicamentos. A polifarmácia foi verificada em 15 idosos. Entre os 117 medicamentos em uso, considerando-se repetições, 13 são inapropriados para idosos, destacando-se diazepam e fluoxetina, cada um utilizado por três idosos. Verifica-se que é imprescindível reduzir o uso de medicamentos inapropriados para melhorar a qualidade de vida dos idosos. É necessária a colaboração do prescritor e do profissional farmacêutico, que é responsável por avaliar a prescrição, identificar riscos relacionados à terapêutica e intervir através da comunicação com o prescritor responsável, fornecendo informações e sugestões que facilitem a utilização de serviços de saúde.

\footnotetext{
Curso de Farmácia, Departamento de Ciências da Saúde. Universidade Regional do Noroeste do Estado do Rio Grande do Sul - UNIJUÍ. Ijuí, Rio Grande do Sul, Brasil.

Correspondência/ Correspondence

Vanessa Adelina Casali Bandeira

Universidade Regional do Noroeste do Estado do Rio Grande do Sul - UNIJUÍ. Rua do Comércio, 3000 - Bairro Universitário

98700-000 - Ijuí, Rio Grande do Sul, Brasil

E-mail: vanessa.acbandeira@yahoo.com.br.
}

Palavras-chaves: Saúde do idoso. Uso de medicamentos. Polimedicação. 
Abstract

The aging process is characterized by several bodily transformations, which can changes the effect of many drugs and be inappropriate to the elderly, due to the lack of efficacy or adverse effects outweigh the benefits. This paper aimed to identify the drugs used by elderly attending the Elderly Care Program (ECP), and investigate the use of potentially inappropriate drugs in this population. It was a quantitative and descriptive study with secondary data collection from medical records/registers of the elderly by ECP (University Extension Project of UNIJUÍ). Among the elderly enrolled, 16 received and / or are receiving drug therapy with pharmaceutical follow-up. The sample comprised 11 women, mean age $75.2 \pm 7.5$ years. Each elderly receives $3.3 \pm 7.3$ drugs on average. Polypharmacy was found in 15 elderly. Among the 117 drugs in use, considering replications, 13 are considered inappropriate for elderly, especially diazepam and fluoxetine, each used by three elderly. It is necessary to reduce the use of inappropriate drugs to improve the quality of life of elderly. This requires the collaboration of the prescriber and pharmacist, who is responsible for evaluating the prescription, identifying risks related to therapeutic intervention and communicate with the prescriber, providing information and suggestions that facilitate the use of health services.
Key words: Health problems. Drug use. Polypharmacy.

\section{INTRODUÇÃO}

O aumento da expectativa de vida é um desejo eminente de todas as sociedades, e uma realidade, inclusive nos países mais pobres. ${ }^{1}$ Entretanto, a longevidade aponta a carência de serviços adequados ao cuidado de idosos, necessitando de medidas urgentes, considerando as especificidades desta população. ${ }^{2}$

O processo de envelhecimento é um conjunto de alterações orgânicas que acarretam modificação da composição corporal, aumento do tecido adiposo e redução das atividades fisiológicas, ${ }^{3}$ alterando a farmacocinética dos medicamentos, em nível de absorção, biodisponibilidade e redução do metabolismo hepático, devido à redução das enzimas metabolizadoras, do fluxo sanguíneo e no tamanho do fígado. ${ }^{4}$ Também ocorrem modificações na função renal, com redução progressiva da taxa de filtração glomerular e no fluxo sanguíneo, associados a mudanças estruturais que incluem a perda de massa renal, ${ }^{5}$ com redução na eliminação renal dos fármacos, proporcional à redução do clearence de creatinina. ${ }^{4}$

Secoli \& Duarte ${ }^{6}$ salientam que a diminuição dos níveis de albumina sérica, característica desta faixa etária, aumenta a fração livre dos agentes farmacológicos no plasma, elevando os índices de toxicidade ao fármaco. Além disso, segundo Michocki, ${ }^{3}$ na população idosa podem ocorrer alterações relacionadas ao receptor, de um tecido ou órgão alvo. Diante do exposto, alguns medicamentos são considerados inadequados para a população idosa, conforme classificação de Beers ${ }^{7}$ atualizada por Fick et al. ${ }^{8}$

Os autores consideram medicamentos inadequados aqueles impróprios para uso em idosos, em comparação a outras categorias, por falta de eficácia terapêutica ou pelo fato de seus efeitos adversos serem superiores aos benefícios. ${ }^{9}$ $\mathrm{O}$ uso desses medicamentos em idosos aumenta o número de reações adversas e se relaciona à piora do estado físico e mental do usuário e maior utilização de serviços de saúde. ${ }^{9}$ Estudo realizado em hospitais americanos, em 1992, concluiu ainda que o idoso é acometido por muitas reações adversas que poderiam ser evitadas. $^{10}$

É importante considerar que a maioria dos idosos, por conta das inúmeras causas de fragilidade e maior vulnerabilidade para o desenvolvimento de doenças crônicas ou agudas, 
utilizam muitos medicamentos e assim estão mais expostos ao risco de reações adversas, o que aumenta quando se utilizam medicamentos inadequados. ${ }^{9}$

Coelho Filho et al. ${ }^{11}$ entrevistaram idosos no Ceará e observaram a utilização de pelo menos um medicamento inadequado em $13,1 \%$ dos idosos da área central, 17,1\% da área intermediária e $18,9 \%$ da área periférica.

A porcentagem de uso inapropriado no estudo acima é agravada pelos dados apontados por Secoli \& Duarte, ${ }^{6}$ que mostram que a população dos Estados Unidos é composta por $12 \%$ de idosos, que utilizam 30\% dos medicamentos prescritos no país, consumindo 40\% daqueles de venda livre. Já na Inglaterra, são $18 \%$ da população e consomem $30 \%$ dos medicamentos, sendo $70 \%$ consumidores daqueles de venda livre.

Tem-se observado alta prevalência de utilização de medicamentos em idosos no Brasil. Em estudo realizado em Santa Rosa-RS, por Flores \& Benvegnú, ${ }^{12}$ identificou-se prevalência de $82 \%$ na semana anterior à entrevista; em Porto AlegreRS, a prevalência foi de $91 \%$ entre os idosos entrevistados ${ }^{13} \mathrm{e}$, ainda, a polifarmácia é comum entre os idosos, favorecendo a ocorrência de efeitos adversos e interações medicamentosas. Segundo Secoli \& Duarte, ${ }^{6}$ os medicamentos podem causar reações indesejadas em qualquer faixa etária, mas sua incidência aumenta com a idade.

Destaca-se que os idosos não são avaliados na maioria dos ensaios clínicos, o que dificulta a extrapolação dos dados para uso crônico de medicamentos. Diante disso, o uso de medicamentos nessa faixa etária é empírico devido à falta de evidências suficientes sobre os benefícios e riscos de sua utilização. ${ }^{9}$

Assim, o objetivo do estudo foi identificar os medicamentos utilizados, investigando o uso de medicamentos potencialmente inapropriados aos idosos assistidos pelo P.A.I. da Universidade Regional do Noroeste do Estado do Rio Grande do Sul (UNIJUÍ), Ijuí-RS.

\section{METODOLOGIA}

Programa de Atenção ao Idoso (P.A.I.)

Trata-se de projeto de Extensão Universitária que envolve acadêmicos e professores dos cursos de Enfermagem, Farmácia, Fisioterapia e Nutrição, do Departamento de Ciências da Saúde da UNIJUÍ.

O P.A.I. busca assistir idosos (idade igual ou maior que 60 anos) de baixa renda residentes na área urbana do município de Ijuí-RS, que apresentem risco médio a alto de internação e/ou reinternação hospitalar, segundo classificação de risco validada por Boult et al. ${ }^{14} \mathrm{O}$ programa iniciou suas atividades em 2008, em 2010 sofreu modificações na forma de atendimento, deixando a busca hospitalar e realizando visitas domiciliares aos idosos cadastrados, visando a acompanhar e contribuir para melhorar as condições de saúde desses idosos.

A participação das acadêmicas e professora do Curso de Farmácia no Programa se dá através do acompanhamento da terapia medicamentosa do idoso, utilizando o Método Dáder ${ }^{15}$ de seguimento farmacoterapêutico, procurando assim identificar o risco de interações medicamentosas potenciais e a não-adesão ao tratamento. A partir dos dados obtidos, são elaboradas estratégias para o cumprimento da farmacoterapia e orientações escritas individualizadas relacionadas ao uso adequado dos medicamentos, dirigidas ao idoso ou seu cuidador.

\section{Estudo e Amostra}

Realizou-se estudo transversal, quantitativo e descritivo, com coleta de dados secundários, a partir de prontuários/cadastros dos idosos atendidos pelo P.A.I. Foram incluídos no estudo os prontuários de todos os idosos cadastrados no Programa, com risco médio a alto de internação e/ ou reinternação e que receberam atendimento farmacoterapêutico de acordo com o Método Dáder, ${ }^{15}$ pela equipe da área da Farmácia no período de janeiro de 2009 a junho de 2010. Foram excluídos aqueles que apresentaram risco baixo, os que faleceram, que não consentiram que seus 
dados pudessem ser usados em estudos quando foram cadastrados ou que não foram mais acompanhados pelo Programa.

\section{Instrumentos e Variáveis}

Analisaram-se os medicamentos em uso na última avaliação de acompanhamento farmacoterapêutico, os quais foram classificados no primeiro e segundo níveis, conforme o sistema de classificação Anatomical Therapeutic Chemical (ATC) do Nordic Council on Medicines, index 2010. ${ }^{16}$

A identificação dos medicamentos inadequados considerou os critérios de Fick et al., ${ }^{8}$ que classificam os medicamentos utilizados em apropriados ou inapropriados de acordo com indicação, eficácia, dose, praticidade, interação fármaco-fármaco, interação fármaco-doença, duplicidade de medicamentos, duração e custo. Considerou-se a polifarmácia como o uso de cinco ou mais medicamentos simultaneamente. ${ }^{6}$

Para fins desta pesquisa, foram considerados todos os medicamentos relatados pelos entrevistados no momento do seguimento farmacoterapêutico, incluídos os medicamentos adquiridos com prescrição e aqueles administrados por automedicação. Os dados foram representados por média, desvio padrão e frequência.

\section{Aspectos Éticos}

Todos os idosos assistidos ou seus cuidadores, em caso de impossibilidade, expressaram consentimento para acompanhamento e assistência pelo P.A.I.

Foram seguidas as recomendações da Resolução CNS n 196/1996 e obtida aprovação do Comitê de Ética em Pesquisa da UNIJUÍ, sob Parecer Consubstanciado ${ }^{\circ} 173 / 2010$, sendo que todos os participantes ou seus responsáveis assinaram o Termo de Consentimento Livre e Esclarecido.

\section{RESULTADOS}

Dos idosos cadastrados no P.A.I., 16 receberam e/ou estão recebendo acompanhamento farmacoterapêutico pela equipe da farmácia, sendo 11 mulheres e cinco homens, com idade média de 75,2 (DP \pm 7,5 anos) (mínimo de 60 e máximo de 87).

O número médio de medicamentos utilizados foi de 7,3 $\pm 3,3$ por idoso, com o uso máximo de 14 medicamentos por um dos pacientes. A classificação dos medicamentos, de acordo com o sistema ATC nível 1 (grupo terapêutico), encontra-se na tabela 1. 
Tabela 1 - Classificação dos medicamentos utilizados pelos idosos segundo $1^{\circ}$ nível da classificação ATC. Ijuí, RS, 2010.

\begin{tabular}{lcc}
\hline Grupo de medicamentos & $\begin{array}{c}\mathrm{N}^{\circ} \\
\text { medicamentos }\end{array}$ & Porcentagem (\%) \\
\hline N - Sistema nervoso & 34 & 29,1 \\
C - Aparelho cardiovascular & 32 & 27,3 \\
A - Trato alimentar e metabolismo & 25 & 21,4 \\
B - Sangue e órgãos hematopoiéticos & 10 & 08,6 \\
M - Sistema músculo esquelético & 06 & 05,1 \\
H - Preparações hormonais sistêmicas, & 05 & 04,3 \\
$\quad$ excluindo hormônios sexuais e insulinas & 03 & 02,6 \\
D - Dermatológicos & 01 & 00,8 \\
J - Antiinfecciosos gerais para uso sistêmico & 01 & 00,8 \\
G - Aparelho genito-urinário e hormônios & & 100,0 \\
$\quad$ sexuais & 117 & \\
Total & &
\end{tabular}

Os medicamentos utilizados em maior número pelos idosos encontram-se no grupo dos que atuam no sistema nervoso e aparelho cardiovascular respectivamente, os quais foram também classificados no nível $2 \mathrm{da}$ ATC, conforme a tabela 2 .

Tabela 2 - Classificação dos medicamentos utilizados pelos idosos segundo $2^{\circ}$ nível de classificação ATC. Ijuí, RS, 2010.

\begin{tabular}{llcl}
\hline $1^{\circ}$ nível ATC & \multicolumn{1}{c}{ 2 $^{\circ}$ nível ATC } & $\mathrm{N}^{\circ}$ med. & $\%$ \\
\hline $\mathrm{N}^{* *}$ & N03 - Antiepiléticos & 11 & 32,4 \\
& N05 - Psicolépticos & 11 & 32,4 \\
& N02 - Analgésicos & 06 & 17,6 \\
& N06 - Psicoanalépticos & 03 & 08,8 \\
& N07- Outros medicamentos para o sistema & 03 & 08,8 \\
& nervoso & 34 & 100 \\
Total & C03 - Diuréticos & 11 & 34,4 \\
C** & C09 - Agentes com ação no sistema renina- & 08 & 25,0 \\
& angiotensina & 04 & 12,5 \\
& C07 - Agentes beta-bloqueadores & 03 & 09,4 \\
& C01 - Terapia cardíaca & 03 & 09,4 \\
& C02 - Anti-hipertensivos & 02 & 06,2 \\
& C10 - Agentes modificadores de lipídeos & 01 & 03,1 \\
& C08 - Bloqueadores dos canais de cálcio & 32 & 100,0 \\
\hline
\end{tabular}

* Sistema nervoso; $*$ Aparelho cardiovascular 
Verificou-se que 15 idosos utilizavam mais de cinco medicamentos, tendo apenas um idoso não polimedicado. Entre os 117 medicamentos em uso, com repetições, foram encontrados 13 inapropriados para idosos, perfazendo em média 0,813 medicamentos inapropriados por idoso. Entre os idosos que não utilizavam medicamentos inapropriados, um utilizava nove medicamentos, outro utiliza oito, dois utilizavam seis e um utilizava um medicamento. Estes idosos apresentaram idades entre 69 e 86 anos.

Diazepam e fluoxetina foram os medicamentos inapropriados mais utilizados, por três idosos cada. Ainda, foi encontrado o uso de doxazosina por dois idosos. Carisoprodol, escopolamina, óleo mineral, bromazepam e clorfeniramina foram utilizados por um idoso cada.

\section{DISCUSSÃO}

A polifarmácia foi identificada em 15 idosos do presente estudo. Além disso, os idosos, devido a suas particularidades, são mais suscetíveis à ação dos fármacos, o que aumenta os problemas relacionados a sua administração e a probabilidade de interação entre os fármacos. ${ }^{3} \mathrm{~A}$ prática da polifarmácia é multifatorial, sendo influenciada sobretudo pelas doenças crônicas e manifestações clínicas resultantes do envelhecimento. ${ }^{17}$ É provável que esses fatores determinem o uso de muitos medicamentos pela maioria dos idosos do P.A.I.

Araújo, Magalhães \& Chaimowicz, ${ }^{18}$ em estudo realizado com idosos de um Programa de Saúde da Família de Belo Horizonte-MG, com prevalência de polifarmácia de $27,7 \%$, mostraram a associação desta com a presença de cinco ou mais problemas de saúde e ao uso de medicamentos inapropriados, sendo que tal prática se reflete no aumento do gasto mensal com medicamentos. Além disso, o perfil dos usuários de polifarmácia, conforme Lassila et al., ${ }^{19}$ é predominantemente composto por idosos do sexo feminino, com idade avançada, residindo na zona rural e com baixo nível de escolaridade.

A polifarmácia é frequente nesta faixa etária: Coelho Filho, Marcopito \& Castelo, ${ }^{11}$ em estudo realizado em Fortaleza-CE, com entrevista domiciliar, encontraram que $13,6 \%$ dos idosos usavam cinco ou mais medicamentos. Dessa forma, a polifarmácia em idosos parece ser uma tendência nacional, confirmada na população de nosso estudo.

Verificou-se que a média de medicamentos utilizados no presente estudo é semelhante ao encontrado por Costa. ${ }^{20}$ No entanto, no que se refere à polifarmácia, a prevalência é maior em relação aos demais estudos, sendo importante considerar que muitos dos idosos atendidos pelo programa apresentavam alto risco de reinternação hospitalar e se encontravam acamados, necessitando do uso de vários medicamentos.

Dentre os fármacos utilizados pelos idosos, os que atuam no sistema nervoso e no aparelho cardiovascular foram os grupos de medicamentos mais utilizados, semelhante ao que foi evidenciado por Correr et al..$^{21} \mathrm{em}$ estudo realizado em Apucarana-PR, com idosos de uma instituição geriátrica e idade média de 67,1 anos: dentre os medicamentos utilizados, $47,8 \%$ atuam no sistema nervoso, seguidos por $36,4 \%$ no aparelho cardiovascular. No entanto, difere do estudo de Coelho Filho, Marcopito \& Castelo, ${ }^{11}$ no qual prevaleceu o uso de medicamentos para o sistema cardiovascular (29,3\%), seguido daqueles para o sistema nervoso $(13,5 \%)$. O mesmo foi evidenciado por Aguiar et al. ${ }^{22}$ em instituições asilares no Nordeste do Brasil, com média de idade de 83,2 anos, onde $47,3 \%$ dos medicamentos utilizados atuam no sistema cardiovascular e $20,1 \%$, no sistema nervoso. Os fármacos que atuam nos sistemas cardiovascular e nervoso provavelmente foram os mais utilizados, pelo fato de as doenças crônico-degenerativas atingirem de modo mais acentuado essa faixa etária.

O consumo elevado de medicamentos contribui para o uso de fármacos considerados inapropriados para idosos. ${ }^{3}$ Dos medicamentos utilizados no presente estudo, $13(11,11 \%)$ são considerados inapropriados para idosos diferindo dos estudos de Costa, ${ }^{20}$ Araújo, Magalhães \& Chaimowicz, ${ }^{18}$ Aguiar et al., ${ }^{22}$ nos quais a utilização de medicamentos considerados 
inapropriados foi de $38,9 \%, 33,5 \%$, e $28,7 \%$, respectivamente. Também nos Estados Unidos foi identificado maior percentual de utilização de medicamentos inapropriados $(21,3 \%)$ no estudo de Zhan et al. ${ }^{23}$ Em pesquisa retrospectiva, nos anos de 1992 e 2000, realizada em um serviço de emergência de um hospital americano, foram observados os medicamentos administrados em idosos de acordo com os critérios de Beers para medicamentos inapropriados, e estes foram utilizados por $12,6 \%$ dos idosos. ${ }^{24}$

Rothberg et al., ${ }^{25}$ em uma coorte retrospectiva do período de setembro de 2002 a junho de 2005 em 348 hospitais americanos, verificaram que $49 \%$ dos pacientes acima de 65 anos receberam pelo menos um medicamento inapropriado, sendo que os médicos cardiologistas representam a maior taxa de prescrição (48\%), enquanto os geriatras têm a menor taxa (24\%). Neste sentido, verificase a necessidade de conhecer as peculiaridades do tratamento ao idoso. Com isso, a menor utilização de medicamentos inapropriados nos idosos assistidos pelo P.A.I. em relação aos demais estudos poder estar relacionada ao tamanho da amostra, o que limita a extrapolação dos nossos resultados e também pode estar relacionado ao atendimento individualizado do P.A.I. aos idosos assistidos pelo programa. O estudo de Pugh et al., ${ }^{26}$ realizado com idosos nos Estados Unidos, mostrou que após uma intervenção da equipe de pesquisa, com ajustes de diagnóstico, dose e duração do tratamento, a prescrição de medicamentos inapropriados diminuiu de $33 \%$ para $23 \%$, demonstrando que ações conjuntas podem melhorar a qualidade da terapêutica nesses indivíduos.

No presente estudo, os medicamentos inapropriados se encontram classificados junto aos demais medicamentos, conforme a ATC. Entre os que atuam no sistema nervoso, citam-se o diazepam e bromazepam, que são pscicolépticos (N05) e fluoxetina, um psicoanaléptico (N06). Entre os que atuam no trato alimentar e metabolismo, estão a escopolamina, um medicamento para distúrbios gastrointestinais funcionais (A02) e o óleo mineral, um laxativo (A06). Também foram utilizados os que atuam no sistema músculo-esquelético, como o carisoprodol, um relaxante muscular (M03), para o sistema cardiovascular, como a doxazosina, um anti-hipertensivo (CO2) e sistema respiratório, a clorfeniramina, um anti-hitamínico para uso sistêmico (R06). Esses medicamentos deveriam ser evitados, uma vez que existem outras alternativas farmacológicas mais indicadas para uso em idosos.

Caterino et al. ${ }^{24}$ verificaram que seis medicamentos corresponderam a 70,8\% dos medicamentos inapropriados utilizados em idosos, sendo, em ordem decrescente: prometazina, meperidina, propoxifeno, hidroxizine, difenidramina e diazepam diferentes dos mais utilizados em nosso estudo, exceto diazepam.

Dentre os medicamentos inapropriados para idosos, destaca-se, em nosso estudo, o uso de diazepam, classificado conforme ATC em seus níveis decrescente como um derivado benzodiazepínico, ansiolítico, psicoléptico que atua no sistema nervoso. Segundo os critérios de Fick et al., ${ }^{8}$ é um benzodiazepínico de longa duração considerado inapropriado por ter longa meia-vida em idosos, produzindo sedação prolongada e aumento do risco de quedas e fraturas - mesmos efeitos aplicados ao uso de bromazepam. O risco de quedas e fraturas foi verificado por Coutinho \& Silva ${ }^{27}$ sobretudo nos usuários de benzodiazepínicos, quando comparados às demais classes terapêuticas. Os autores recomendam para idosos o uso de benzodiazepínicos de ação curta e intermediária. ${ }^{8}$ Entretanto, estudo de caso-controle realizado dentro do estudo de coorte do estudo de Hooft et $a{ }^{28}$ verificou que não foi significativa a diferença de fratura entre os usuários de benzodiazepínicos inapropriados e os considerados apropriados, mas que o maior risco de fratura está relacionado a doses elevadas e tempo de uso prolongado, independentemente do benzodiazepínico utilizado.

No entanto, os benzodiazepínicos de longa duração são utilizados com frequência. No estudo de Costa, ${ }^{20} 38,9 \%$ dos idosos internados utilizavam pelo menos um medicamento 
inapropriado, dos quais o diazepam foi o mais encontrado (14,0\% dos 157 medicamentos inapropriados utilizados, considerando repetições). O alto risco relacionado ao uso desse medicamento preocupa e alerta para necessidade de intervenção, visto que foi o mais utilizado pelos idosos do P.A.I., em conjunto com a fluoxetina e considerando que ambos apresentam risco alto, conforme determinam Fick et al. ${ }^{8}$

Cruz et al. ${ }^{29}$ verificaram que o uso prolongado de diazepam em idosas desencadeou efeitos adversos como angústia, dores articulares, depressão e tontura, o que pode elevar os riscos de dependência e tolerância a esses fármacos. Diante disso, alertam que o uso indiscriminado de benzodiazepínicos pode desencadear efeitos adversos desnecessários e interações medicamentosas, expondo assim os usuários a riscos também desnecessários.

A fluoxetina, que segundo a classificação ATC, é um inibidor seletivo da recaptação da serotonina, com ação antidepressiva e psicoanaléptica e atuando no sistema nervoso, tem sua efetividade questionável nas doses toleradas por idosos. Por isso, esse medicamento deve ser evitado (especialmente para uso prolongado), pois apresenta longa meia-vida, podendo ocasionar estimulação exagerada do sistema nervoso central, distúrbios do sono e agitação. Apesar de existirem alternativas mais seguras com a mesma atividade terapêutica, ${ }^{8}$ verificou-se que três idosos atendidos pelo P.A.I. utilizavam o medicamento, expondose aos riscos inerentes ao uso dessa substância.

A doxazosina tem potencial para causar efeitos como hipotensão, boca seca e problemas urinários, enquanto o carisoprodol e a clorfeniramina são inadequados devido aos potentes efeitos adversos anticolinérgicos, como sedação e fraqueza. $\mathrm{O}$ óleo mineral também é inapropriado devido ao grande potencial para aspiração e efeitos adversos, para o qual existem alternativas mais seguras. ${ }^{8}$ Por causa dessas inadequações aos idosos, tais medicamentos devem ser evitados ao máximo nesta faixa etária, o que precisa ser alertado aos prescritores e dispensadores. No entanto, quando o uso é necessário, deve ser realizado com cautela, acompanhamento e monitoração apropriados. ${ }^{8}$
A utilização de polifarmácia e o uso de medicamentos inapropriados tornam os idosos mais suscetíveis a morbidade, mortalidade e utilização de recursos do serviço de saúde. ${ }^{30}$ Além disso, a farmacoterapia dos idosos exige cuidados individualizados, considerando múltiplas doenças, associadas a alterações fisiológicas que podem interferir na farmacocinética $e$ farmacodinâmica dos medicamentos utilizados. ${ }^{31}$

Também foi evidenciado em nosso estudo que os idosos que não utilizavam medicamentos inapropriados estavam expostos a interações medicamentosas em potencial, pois dentre os cinco, quatro eram polimedicados, favorecendo tal ocorrência. Assim, se por um lado esses idosos não estão expostos ao uso de medicamentos inadequados, por outro, precisam de cuidado farmacoterapêutico, visto que interações também podem refletir de forma negativa sobre sua saúde.

Segundo Bueno et al., ${ }^{32}$ os idosos acompanhados pelo P.A.I. no período de março de 2008 a julho de 2009 utilizaram uma média de $5,2 \pm 3,7$ medicamentos, verificando-se que estavam expostos a, em média, quatro interações medicamentosas. O estudo ressaltou a importância de o profissional prescritor conhecer a influência que um fármaco pode exercer sobre o outro e o papel do farmacêutico como o profissional capaz de identificar possíveis interações e, em conjunto com os demais profissionais de saúde, especialmente com o prescritor, poder intervir quando necessário. ${ }^{32}$ Percebe-se, assim, que os idosos assistidos pelo P.A.I. estão expostos a um considerável número de interações, possivelmente pelo fato de serem polimedicados.

Nesse sentido, é imprescindível a atuação de profissionais da saúde qualificados, sobretudo quando as atividades são direcionadas ao processo saúde/doença, com o objetivo de melhorar a qualidade de vida, atuando na integralidade das ações visando a promoção, prevenção, cura e reabilitação das condições de saúde. ${ }^{33}$ Cabe aos profissionais que assistem idosos identificar e compreender suas limitações, educar e orientar os cuidadores e adotar esquemas terapêuticos 
simples, ou o mais simples possível, minimizando efeitos adversos. ${ }^{6}$

A atuação de uma equipe multiprofissional facilita o cuidado do paciente idoso, desenvolvendo diversas ações voltadas ao acompanhamento de suas necessidades individuais, através de uma visão multiprofissional que auxilia na tomada de decisão para a elaboração do plano terapêutico. Além disso, conforme a revisão de Quinhalha \& Correr ${ }^{34}$ sobre as listas de medicamentos inadequados existentes, os profissionais devem considerar como limitação a intensa exclusão e inclusão de medicamentos no mercado, o que as torna rapidamente desatualizadas.

O aconselhamento e o acompanhamento do uso dos medicamentos são de extrema importância para a população idosa, devido a suas múltiplas doenças, com terapias diferenciadas e uso concomitante de muitos medicamentos. ${ }^{33} \mathrm{O}$ profissional farmacêutico pode auxiliar nos principais problemas encontrados com o uso de medicamentos nessa população, como identificar medicamentos inapropriados, a presença de interações, reações adversas, automedicação, administração incorreta e, com isso, fornecer as informações necessárias sobre a doença e os medicamentos prescritos, garantindo a segurança $\mathrm{da}$ terapia prescrita. ${ }^{34}$ Desse modo, através da atenção farmacêutica, na qual o profissional assume papel ativo em benefício do paciente, se estabelece uma relação farmacêutico-pacientemedicamento com atividades voltadas para o autocuidado, promovendo melhora na qualidade de vida dos idosos. ${ }^{35}$

\section{CONCLUSÃO}

O uso de medicamentos inapropriados para idosos se faz ainda presente na população estudada e é realidade na população brasileira. Para reduzir a utilização de tais medicamentos e sua repercussão negativa na saúde do idoso, é necessário que prescritores e dispensadores conheçam os medicamentos que devem ser evitados e façam o acompanhamento necessário quando seu uso for imprescindível.

A amostra do estudo foi pequena, o que limitou inferências gerais à população. Assim, encontrou-se a utilização de menos medicamentos inapropriados, quando comparada a outros estudos, o que pode estar relacionado a intervenção farmacêutica junto aos idosos assistidos pelo P.A.I., sugerindo a importância dessa atuação, assim como sua ampliação, para aprimorar o acompanhamento.

O farmacêutico é o profissional responsável pela avaliação da prescrição, podendo, nesse momento, identificar riscos relacionados a terapêutica e intervir, através da comunicação com o prescritor responsável, trocando informações e sugestões que possam reduzir a ocorrência de reações adversas a medicamentos e a necessidade de utilização de serviços de saúde.

A atuação farmacêutica, na qual o profissional assume papel ativo em benefício do paciente, auxiliando o prescritor na seleção do medicamento, colabora diretamente para alcançar o tratamento desejado. O uso de muitos medicamentos, mesmo que apropriados, exige o acompanhamento farmacoterapêutico.

Os idosos que utilizam medicamentos inapropriados têm maiores possibilidades de apresentar as reações relacionadas ao uso desses medicamentos. Sendo assim, é importante que o prescritor e o farmacêutico conheçam os medicamentos inapropriados, para evitar os problemas decorrentes de sua utilização indevida em idosos. 


\section{REFERÊNCIAS}

1. Veras R. Envelhecimento populacional contemporâneo: demandas, desafios e inovações. Rev. Saúde Pública 2009; 43 (3): 548-54.

2. Veras RLR,et al. Novos paradigmas do modelo assistencial no setor saúde: conseqüência da explosão populacional dos idosos no Brasil. In: Veras RP. Terceira idade: gestão contemporânea em saúde. Rio de Janeiro: Relume Dumara; 2002. p. 11-79.

3. Michocki RJ. Polifarmácia e princípios da terapia com drogas. In: Adelman AM, Daly MP. Geriatria. Rio de Janeiro: Revinter Ltda; 2004. p. 65-76.

4. Cusack BJ. Pharmacokinetics in older persons. Am J Geriatr Pharmacother 2004; 2 (4): 274-302.

5. Weinstein JR, Anderson S. The Againg Kidney: physiological changes. adv chronic kidney dis 2010; 17 (4): 302-307.

6. Secoli SR, Duarte YAO. Medicamentos e a assistência domiciliária. In: Duarte YAO, Diogo MJD. Atendimento domiciliar: um enfoque gerontológico. São Paulo: Atheneu; 2000. p. 326-35.

7. Beers MH. Explicit criteria for determining potentially inappropriate medication use by the elderly. Arch Intern Med 1997; 157(2): 1531-36.

8. Fick DM, et al. Updating the beers criteria for potentially inappropriate medication use in older adults. Arch Intern Med 2003; 163(2): 2716-52.

9. Flores LM, Colet CF. Riscos da polifarmácia em clientes idosos. In: Malagutti W, Bergo AMA. Abordagem interdisciplinar do idoso. Rio de Janeiro: Rubio; 2010. p. 291-302.

10. Thomas EJ, Brennan TA. Incidence and types of preventable adverse events in elderly patients: population based review of medical records. BMJ 2000 ; 320 (7237): 741-4.

11. Coelho Filho JM, Marcopito LF, Castelo A. Perfil de utilização de medicamentos por idosos em área urbana do Nordeste do Brasil. Rev de Saúd Públic 2004; 38 (4): 557-64.

12. Flores VB, Benvegnú LA. Perfil de utilização de medicamentos em idosos da zona urbana de Santa Rosa. Cad. Saúde Pública 2008; 24(6): 1439-46.

13. Flores LZ, Mengue SS. Uso de medicamentos por idosos em região do sul do Brasil. Rev. Saúde Pública 2005; 39(6): 924-9.

14. Boult L, Boult C, Pirie P, Pacala JT. Test-retest reliability of a questionnaire that identifies elders at risk for hospital admission. J Am Geriatr Soc 1994 ; 42 (7): 707-11.
15. Machuca M, Fenández-LLimós F, Faus MJ.

Método Dáder :guía de seguimiento fármacoterapéutico. 2003. Disponível em: URL http://www.crf-ba.org.br/biblioteca/ manual_dader_at_farmaceutica.pdf.

16. World Health Organization. Collaborating Centre for Drug Statistics Methodology. Anatomical Therapeutic Chemical ATC/DDD Index 2009. Oslo: World Health Organization; 2009. Disponível em: URL http:// www.whocc.no/atcddd/.

17. Secoli SR. Polifarmácia: interações e reações adversas no uso de medicamentos por idosos. Rev. Bras. Enferm. 2010; 63 (1): 136-40.

18. Araújo CMC, Magalhães SMS, Chaimowicz F. Uso de Medicamentos Inadequados e Polifarmácia entre Idosos do Programa Saúde da Família. Lat. Am. J. Pharm. 2010; 29 (2): 178-84.

19. Lassila HC, et al. Use of prescription medications in an elderly rural population: the movies Project. Ann Pharmacother 1996; 30(6): 589-95.

20. Costa SC. Avaliação da prescrição de medicamentos para idosos internados em serviço de clínica médica do sistema único de saúde em um hospital público universitário brasileiro .Belo Horizonte. Dissertação[ Mestrado em Ciências da Saúde]-Universidade Federal de Minas Gerais; 2009. Disponível em: URL http://hdl.handle.net/1843/ECJS7YYHUA.

21. Correr CJ, et al. Riscos de problemas relacionados com medicamentos em pacientes de uma instituição geriátrica. Rev. Bras. Cienc. Farm. 2007; 43 (1): 55-62.

22. Aguiar PM, et al. Avaliação de farmacoterapia de idosos residentes em instituição asilares no nordeste do Brasil. Lat. Am. J. Pharm. 2008; 27 (3): 454-59.

23. Zhan C, et al. Potentially Inappropriate Medication Use in the Community-Dwelling Elderly. JAMA 2001; 286 (22): 2823-9.

24. Caterino JM, Emond JÁ, Camargo CA. Inappropriate Medication Administration to the Acutely Ill Elderly: a nationwide emergency department study. J Am Geriatr Soc. 2004; 52(11): 1847-55.

25. Ruthberg MB, et al. Potentially inappropriate medication use in hospitalized elders. J Hospital Medicine 2008; 3(2): 91-102.

26. Pugh MJV,et al. Potentially inappropriate prescribing in elderly Veterans: are we using the wrong drug, wrong dose, or wrong duration? J Am Geriatr Soc 2005; 53(8):1282-89. 
27. Coutinho ESF, Silva SD. Uso de medicamentos como fator de risco para fratura grave decorrente de queda em idosos. Cad. Saúde Pública 2002; 18 (5): 1359-66.

28. Hooft CS, et al. Inappropriate benzodiazepine use in older adults and the risk of fracture. British J Clinical Pharmacol 2008; 66 (2): 276-282.

29. Cruz AV,et al. Uso crônico de diazepam em idosos atendidos na rede pública em Tatuí-SP. Rev. Ciênc. Farm. Básica Apl. 2006; 27 (3): 259-67.

30. Hamilton EH, Gallagher PF, O'Mahony D. Inappropriate prescribing and adverse drug events in older people. BMC Geriatrics 2009; 9 (5): 1-4.

31. Pereira SRM. Farmacoterapia Geriátrica. In: Silva, P. Farmacologia. 6. ed. Rio de Janeiro: Guanabara Koogan; 2002. p. 1220-25
32. Bueno CS, et al. A. Utilização de medicamentos e risco de interações medicamentosas em idosos atendidos pelo Programa de Atenção ao Idoso da Unijuí. Rev Ciênc Farm Básica Apl. 2009; 30 (3): 331-8.

33. Marin MJS, Marques APMF, Feres BOM, Saraiba AKH, Druzian S. A atenção à saúde do idoso: ações e perspectivas dos profissionais. Rev. Bras. Geriatr. Gerontol. 2008; 11 (2): 245-58.

34. Quinalha JV, Correr CJ. Instrumento para avaliação da farmacoterapia do idoso: uma revisão. Rev. Bras. Geriatr. Gerontol. 2010; 13 (3): 487-99.

35. Andrade MA, Silava MVS, Freitas O. Assistência Farmacêutica como estratégia para o uso racional de medicamentos em idosos. Cad. Saúde Pública 2004; 25 (1): 55-63. 
